since it delays conduction through the normal tissues only; in such a case amiodarone would be appropriate. In atrial fibrillation associated with hyperthyroidism digitalis may be ineffective and a $\beta$ blocker preferred. And with little evidence that digitalis is of value in preventing attacks of paroxysmal atrial fibrillation quinidine would be first choice.

Secondly, in ventricular arrhythmias the choice is from a larger range of drugs of broadly similar efficacy. In acute ventricular tachyarrhythmias lignocaine is still widely preferred as first choice, with intravenous amiodarone being given if lignocaine fails. Refractory arrhythmias may require other drug treatment: it would make sense, in the absence of other guidance, to substitute a drug of class Ia (for example, procainamide) and then one of class Ic (for example, flecainide). For chronic ventricular arrhythmias a drug of class Ia would be first choice. Amiodarone, while highly effective $^{\gamma}$ and fashionable, has long term adverse effects which are frequent and may be serious, ${ }^{9}$ and its future use seems likely to be only short term, except in selected cases where the benefit is thought to outweigh the long term risks. Of other established drugs, such as mexiletine, none has any clear cut advantages, and it is too soon to say whether tocainide and the class Ic drugs such as flecainide will prove to be any better. ${ }^{10}$

The precise choice of drug in cases such as these may be further influenced by contraindications (anticipated adverse effects or interactions)-for example, disopyramide and quinidine (because of their anticholinergic actions) in patients with glaucoma or prostatism; drugs of class Ia in patients with cardiac failure or heart block (which they will tend to exacerbate); and combinations of drugs which prolong the $\mathrm{Q}-\mathrm{T}_{\mathrm{c}}$ interval (drugs of class Ia and amiodarone, because of the risk of ventricular tachyarrhythmias, particularly torsade de pointes ${ }^{11}$ ).

Further guidance may be given by the technique of inducing a patient's arrhythmia (for example, by rapid pacing or by delivering precisely timed stimuli during the cardiac cycle) and then observing its response to selected drugs. The responsiveness to class I drugs in these controlled circumstances has been claimed to be a good predictor of the subsequent responsiveness during long term treatment for both ventricular and supraventricular tachyarrhythmias, ${ }^{12}$ and in some series this method has predicted effective treatment in over $70 \%$ of patients, ${ }^{13} 14$ though in the case of amiodarone its value is controversial. ${ }^{15}$ There are, however, disadvantages: the technique requires special skill and is not universally available; only a few drugs can be studied at a time; the induced arrhythmia may not prove to be the same as that requiring treatment; and the induction of arrhythmias may be hazardous (in one series just over half of patients required DC cardioversion to terminate the induced arrhythmia, though none $\left.\operatorname{died}^{13}\right)$. Finally, the successful long term suppression of arrhythmias will depend on the achievement of similar plasma concentrations to those effective during the short term study. Because of these problems this technique is likely to be used only in selected patients in skilled centres.

Despite large advances in our understanding of the pathogenesis of cardiac arrhythmias ${ }^{16}{ }^{17}$ and of the pharmacological effects of antiarrhythmic drugs ${ }^{518}$ drug treatment of cardiac arrhythmias remains, if not entirely unsatisfactory, far short of ideal. In most studies a single drug has been given, often to patients thought (though not always proved) to be resistant to other treatment, and has been shown to be effective in a variable proportion of cases. In fewer instances controlled comparisons have been made of two drugs, and even then it has been difficult to determine any features (relating to the patient or his disease) which would lead to a rational preference of one drug to another. Until such information is available we shall continue to depend mostly on empiricism and the avoidance of adverse effects and interactions.

Clinical Reader (Wellcome Lecturer) and Honorary

J K ARonson

Consultant in Clinical Pharmacology,

MRC Unit and University Department of Clinical Pharmacology,

Radcliffe Infirmary

Oxford OX2 6HE

1 Withering W. An account of the foxglove and some of its medical uses: with practical remarks on the dropsy and other diseases. Birmingham: GGJ and J Robinson, 1785 .

2 Wenckebach KF. Die unregelmässige Herztätigkeit und ihre klinische Bedeutung. Leipzig: W Engelmann, 1914.

3 Frey W. Über Vorhofflimmern beim Menschen und seine Beseitigung durch Chinidin. Berliner Klinische Wochenschrift 1918;55:417-9.

4 Frey W. Über Vorhofflimmern beim Menschen und seine Beseitigung durch Chinidin. Berliner Klinische Wochenschrift 1918;55:450-2.

5 Vaughan Williams EM. A classification of antiarrhythmic actions reassessed after a decade of new drugs. F Clin Pharmacol 1984;24:129-47.

6 Mason DT, deMaria AN, Amsterdam EA, Zelis R, Massumi RA. Antiarrhythmic agents I mechanisms of action and clinical pharmacology. Drugs 1973;5:261-91.

7 Touboul P, Attalah G, Gressard A, Michelson G, Chatelain MT, Delahaye JP. Effets électrophysiologiques des agents antiarhythmiques chez l'homme. Tentative de classification. Arch Mal Coeur 1979;72:72-8

8 Rosenbaum MB, Chiale PA, Haedo A, Lázzari JO, Elizari MV. Ten years of experience with amiodarone. Am Heart f 1983;106:957-64

9 Aronson JK. Cardiac glycosides and drugs used in dysrhythmias. In: Dukes MNG, ed. Mevler's side effects of drugs. 10th ed. Amsterdam: Elsevier, 1984:229-316.

10 Aronson JK. Cardiac glycosides and drugs used in dysrhythmias. In: Dukes MNG, ed. Meyler's side effects of drugs annual 7. Amsterdam: Excerpta Medica, 1983:193-209.

11 Krikler DM, Curry PVL. Torsade de pointes, an atypical ventricular tachycardia. Br Heart $\mathrm{J}$ 1976;38:117-20.

12 Fisher JD, Cohen M, Mehra R, Altschuler H, Escher DJW, Furman S. Cardiac pacing and pacemakers II. Serial electrophysiologic-pharmacologic testing for control of recurrent tachyarrhythmias. Am Heart $\mathcal{F}$ 1977;93:658-68.

13 Mason JW, Winkle RA. Electrode-catheter arrhythmia induction in the selection and assessment of antiarrhythmic drug therapy for recurrent ventricular tachycardia. Circulation 1978;58: 971-85.

14 Horowitz LN, Josephson ME, Farshidi A, Spielman SR, Michelson EL, Greenspan AM. Recurrent sustained ventricular tachycardia. 3. Role of the electrophysiologic study in selection of antiarrhythmic regimens. Circulation 1978;58:986-97.

15 Horowitz LN, Spielman SR, Greenspan AM, Webb CR, Kay HR. Ventricular arrhythmias: use of electrophysiologic studies. Am Heart $\mathcal{F}$ 1983;106:881-6.

16 Wit AL, Rosen MR. Pathophysiologic mechanisms of cardiac arrhythmias. Am Hearl f 1983;106: 798-81

17 Zipes DP, Heger JJ, Prystowsky EN. Pathophysiology of arrhythmias: clinical electrophysiology. Am Heart J 1983;106:812-28.

18 Rosen MR. Wit AL. Electropharmacology of antiarrhythmic drugs. Am Heart $\mathcal{f}$ 1983;106:829-39.

\section{Containing the use of diagnostic} tests

In the past 25 years requests received in diagnostic departments have risen by about $10 \%$ a year while inpatient admissions and outpatient attendances have increased by less than $2 \%$ yearly. ${ }^{1-3}$ Much of this inflation is due not to the introduction of new tests but to a greater demand for well established procedures. In the Public Health Laboratory Service, for example, 1.5 million urine specimens were received for culture in 1979 compared with only 0.5 million 11 years earlier. ${ }^{1}$ Reporting on the substantial increase in the use of diagnostic services during the 1960s, Ashley et al commented that "demand cannot continue indefinitely at the present rate," $"$ but more than a decade later demand is still increasing, with diagnostic departments now accounting for over $9 \%$ of the costs of acute hospital services.

Are the current levels of testing justified? We are not sure. Making diagnoses is at the nub of clinical practice, but research on diagnostic tests has concentrated more on issues such as the precision of results obtained in the laboratory and the comparative accuracy of one test against another than on determining which patients benefit from which tests. Results of the few studies which have evaluated the use of diagnostic tests in the NHS suggest that unnecessary investigation is commonplace . $^{5.9}$ After a series of multicentre studies of radiological procedures the Royal College of Radiologists has 
formulated clinical guidelines for their use which, if implemented, would give substantial savings. ${ }^{5}$ Surveys in outpatient clinics ${ }^{67}$ and of emergency admissions to medical wards $^{89}$ have shown that fewer than $10 \%$ of investigations produced results considered to be important in clinical management.

What is the explanation for this tendency among doctors to perform too many tests? Occupational rituals and attitudes within the profession undoubtedly contribute. Doctors, especially junior staff, may perceive the results of diagnostic tests as more "scientific" (and hence credible) and less prone to error than evidence from a history and physical examination. Often house officers make requests to avoid the rebuke of consultants when a test is omitted; by contrast consultants rarely complain about unnecessary investigations. Certain patterns of requesting become the norm with pressure on house officers to be complete instead of performing discriminatory tests geared to the needs of each patient. Furthermore, a patient's attendance at hospital is usually seen as an opportunity to apply a battery of screening tests, many of which are of doubtful value.

What might be done to reduce overinvestigation and encourage a more discriminating use of diagnostic tests? More research is not the only answer-indeed, it might allow another 10 years of rapid growth. We also need to consider strategies for reducing tests which can be applied now. Such strategies may be grouped under four main headings: feedback of information; financial incentives; decision aids; and control by diagnostic departments.

Providing doctors with statistics on the numbers and costs of tests requested by themselves and clinical colleagues is unlikely to have a large effect. ${ }^{1011}$ When combined with education on the appropriate use of tests, statistical feedback may be successful, but doctors may become immune to the statistics with the result that testing returns to preintervention levels. ${ }^{2}$ Regular review of medical records by consultants and junior staff may be a more effective method of modifying clinical practice than the feedback of statistics. In a controlled trial in a teaching hospital in Boston a group of medical residents participating in a weekly chart review ordered $47 \%$ fewer laboratory tests during the year of the study. ${ }^{12}$ Even when the strategy was withdrawn use continued at a lower level than in the control group. This sustained effect was attributed to repeated exposure of junior staff to information about the efficient use of tests-and to the attitudes of senior doctors participating in the chart reviews.

Financial incentives were also examined in this controlled trial. ${ }^{13}$ A group of residents received a reward according to the size of their reduction in use of tests, but they were less successful in achieving economies than the chart review group. Indeed, for some doctors the incentive appeared to cause considerable conflict; for others, a reward in the form of a medical book token may not have been much of an incentive. In the NHS personal rewards to reduce clinical activity may not be acceptable, but financial incentives in the form of money available to spend on medical equipment and other clinical facilities might be provided within the framework of clinical budgeting. In one of the earliest trials of clinical budgeting, conducted in the Westminster Hospital, wards allocated budgets and permitted to redeploy financial savings reduced their use of several diagnostic services-for example, in one ward the costs of bacteriological tests fell by $56 \%$ and in another those of immunological tests fell by $67 \% .^{14}$

If the appropriate use of tests can be stated in the form of guidelines or specific protocols (such as clinical algorithms) these can be applied as decision aids at the point of request. A simple form of decision aid is a notice displayed prominently in locations where request forms are completed. Alternatively, forms may be restructured to "guide and teach" the clinician when ordering tests. ${ }^{15}$ Restructuring may affect use, however, simply because of a change in the size and complexity of the form: those with check lists are administratively efficient but encourage overuse, while those requiring handwritten details (including justification for the request) inhibit use. ${ }^{15}$ Potentially computer based information systems can influence the ordering of tests. In one Birmingham hospital house officers enter patients' problems into a computer, which then prints out, according to a predetermined proto$\mathrm{col}$, recommendations for clinical management. This has led to a modest decrease in use of unnecessary tests and laboratory costs. ${ }^{16}$

Diagnostic departments may also take steps to ensure appropriate use. If protocols have been agreed with clinical consultants and a request is received which does not adhere to a protocol the department can contact the requesting clinician for an explanation. This process has been shown to reduce substantially requests for preoperative cross matching and the use of blood during surgery. ${ }^{17}$ Alternatively, in some circumstances the department may follow a protocol irrespective of the request made by a clinician. For example, in investigating patients with thyroid disease some clinical chemistry laboratories perform only a staged and sequential series of tests-a strategy which has led to a reduction in use of tests in hospitals in British Columbia. ${ }^{18}$ If acceptable to clinicians, diagnostic departments may also limit the number of tests performed on each patient (except in an emergency). Restricting patients in one hospital in the United States to a maximum of eight haematological and clinical chemistry tests daily resulted in a two thirds reduction in their use. ${ }^{19}$

Given this myriad of possible interventions, what might consultants do to reduce investigations? Unfortunately, few comparative intervention studies have been carried out in Britain; we await the results of those in progress. For the consultant who wants to get on and do something now with minimal administrative fuss the weekly chart review with junior staff is probably the best approach. Reducing the use of diagnostic tests throughout a hospital will require the development of guidelines or protocols approved by appropriate divisions and medical executive committees. Implementing and sustaining reductions in use until they become the norm may require multiple interventions, probably best controlled by a small review committee of representatives from each division. The most effective way in the long term, however, of encouraging the efficient use of diagnostic tests is to give doctors more responsibility for the management of clinical resources within the NHS. If savings can be redeployed wisely clinical budgeting must surely provide the key.

F G R Fowkes

Senior Lecturer in Epidemiology and Medical Care,

University of Wales College of Medicine

Cardiff CF4 4XN

\footnotetext{
Morris CA. Trends in microbiology work-loads in the National Health Service: England and Wales (1968-79). Health Trends 1981;13:8-13.

2 Fleming PR, Zilva JF. Work-loads in chemical pathology: too many tests? Health Trends 1981;13: 46-9.

3 W'righton RJ, Oliver RM. Trends in radiological practice in the NHS. Health Trends 1980;12: $21-4$

4 Ashley JSA, Pasker P, Beresford JC. How much clinical investigation? Lancet 1972;i:890-3.

5 Roberts CJ. The effective use of diagnostic radiology. I $R$ Coll Physicians Lond 1984;18:62-5.

6 Hampton JR, Harrison MJG, Mitchell JRA, Prichard JS, Seymour C. Relative contributions of history-taking, physical examination, and laboratory investigation to diagnosis and management of medical outpatients. Br Med $\mathcal{F}$ 1975;ii:486-9.
}

7 Sandler G. Costs of unnecessary tests. Br Med f 1979;ii:21-4. 
8 Stilwell JA, Young D, Cunnington DA. Evaluation of laboratory tests in hospitals. Ann Clin Biochem 1980;17:281-6.

9 Sandler G. Do emergency tests help in the management of acute medical admissions? Br Med $\mathrm{F}$ 1984;289:973-7.

10 Cohen DI, Jones P. Littenberg B, Neuhauser D. Does cost information availability reduce physician test usage? Med Care 1982;20:286-92.

1 Grivell AR, Forgie HJ, Fraser CG, Berry MN. Effect of feedback to clinical staff of information on clinical biochemistry requesting patterns. Clin Chem 1981:27:1717-20.

12 Rhyne RL, Gehlbach SH. Effects of an educational feedback strategy on physician utilisation of thyroid function panels. I Fam Pract 1979;8:1003-7.

3 Martin AR, Wolf MA, Thibodeau LA, Dzau V, Braunwald E. A trial of two strategies to modify the test-ordering behavior of medical residents. N Eingl f Med 1980;303:1330-6.

14 Wickings I. Putting it together. The patient, the purse, and the practice. Lancet 1977;i:239-40.

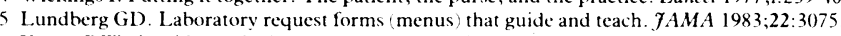

16 Young DW. An aid to reducing unnecessary investigations. Br Med f 1980;281:1610-1.

7 Mintz PD, Lauenstein K, Hume J, Henry JB. Expected hemotherapy in elective surgery. A follow up. 7AMA 1978;239:623-5

18 Hardwick DF, Morrison JI, Tvdeman J, Cassidy PA, Chase WH. Structuring complexity of testing: a process orientated approach to limiting unnecessary laboratory use. Am f Med Technol 1982:48:605-8.

19 Dixon RH, Laszlo J. Utilisation of clinical chemistry services by medical house staff. Arch Intem Med 1974:134:1064-7.

\section{Electromagnetic induction of bone?}

Pulsed electromagnetic treatment of ununited fractures has been employed in over 11000 patients world wide (A A J Goldberg et al, Bioelectrical Repair and Growth Society, second annual meeting, 1982), but the clinical value of this technique remains open to question. It has a long history. In 1853 a report appeared in the United States Medical Times and Gazette describing the successful use of electrical treatment for an ununited fracture, but this was largely ignored in conventional medical circles and dismissed as yet another example of nineteenth century quackery. ${ }^{1}$ Over 100 years later reports began to appear suggesting that the effects of electrical activity on bone formation required further study. ${ }^{2}$

The body and its individual cells are constantly subjected to electromagnetic forces-partly endogenous, from, for instance, the myocardium; and partly external forces, such as the earth's magnetic field and high tension cables. Bone itself develops electrical charge under varying load conditions. Attempting to bend a long bone causes a build up of positive charge on the convex or tensile surface and a transient negative charge on the compressed or concave surface. ${ }^{3}$ Wolff's law states that bone subjected to compression becomes stronger, and it has been widely suggested that electrical phenomena underlie the physiology of adaptive remodelling, though the direct evidence for this is scant. ${ }^{4}$

The formation of new bone has been found with the use of tetracycline labelling, close to areas where a steady electrical negative charge is maintained ${ }^{5}$; and studies of rabbit fibulas showed formation of bone near the negative electrode when a current of between 5 and $20 \mu \mathrm{A}$ was passed across a fracture. ${ }^{6}$ When this current was increased to $100 \mu \mathrm{A}$ necrosis of the tissue was observed, particularly close to the positive electrode. ${ }^{7}$ One criticism of research using implanted electrodes is that an invasive procedure might itself stimulate new bone formation. It has also been argued that "ions" may leak from the electrodes and polarisation occur, producing electrolytic biproducts. ${ }^{8}$ Electromagnetic induction using two external electrodes, electrically insulated from the patient, was employed to overcome these criticisms; with chick tibia as the model, chondrogenesis was found to be inhibited and calcification stimulated. ${ }^{9}$

Numerous explanations were produced for the observed effects in rabbit fibula, chick tibia, and the metatarsal of the weanling mouse. Depletion of oxygen near the negative electrode, increased $\mathrm{pH}$ due to the production of hydroxyl radicals, or increased cyclic adenosine monophosphate concentrations in intact tissues were reported, ${ }^{10}$ though this last has been disputed. "At times these hypotheses were accepted as proved theories, and on the basis of 14 uncontrolled clinical trials on 595 patients with a wide variety of conditions claims were made that electromagnetic treatment resulted in " $50 \%$ faster healing," "increased bone growth," "more stability at two weeks," and " $30 \%$ less time to stability." 12 Success rates claimed for electrical treatment of ununited fractures ranged from $72 \cdot 5 \%^{13}$ and $92 \cdot 5 \%{ }^{14}$ using direct current with implanted electrodes to $80 \%$ using pulsed electromagnetic fields. ${ }^{15}$ A success rate of $93 \%$ was reported when this treatment was combined with bone grafting. ${ }^{16}$ When failure did occur it was thought to result from "inadequate electricity, synovial pseudoarthrosis or infection, or dislodgement of the electrodes." Each series emphasised the importance of "proper cast immobilisation using a nonweight bearing plaster," ${ }^{10}$ but only one author went so far as to state that, "mechanical fixation and bone grafting proved more critical for successful union than did the electrical stimulation," and he continued, "electrical stimulation does not compensate for inadequate fracture fixation." 17

These clinical studies have highlighted three topics of uncertainty. The first is the definition of "non-union" of a fracture. That "non-union exists when the surgeon considers that the fractures will not heal without surgical intervention" is clearly open to question, ${ }^{18}$ but so also is the definition of "no progressive healing over a three month period" 13 when oblique radiographs have not been taken. ${ }^{14} \mathrm{~A}$ tenth of the fractures which Connolly considered initially to be ununited healed spontaneously without further treatment. ${ }^{17} \mathrm{He}$ argued that pain, mobility of the fracture on fluoroscopy, intraosseous phlebography, ${ }^{1920}$ and bone scintigraphy ${ }^{21}$ were important in deciding which patients to include in the study. The mere radiological persistence of a fracture line does not invariably indicate non-union.

The second difficulty has arisen through the use of historical controls. By definition, an ununited fracture should remain ununited, unless modified by, for instance, the introduction of bone graft. Grafting procedures are reported to produce a union rate of between $85 \%$ and $98 \%$ at the first attempt, dependent on the severity and site of the nonunion $^{22}$ and the grafting technique. ${ }^{23}$ This figure falls to $66 \%$ with the second grafting and $64 \%$ at the third attempt. ${ }^{24}$ But without grafting $12.5 \%$ of fractures of the tibial shaft of 12 months' duration still unite with conservative management alone..$^{25}$ In a series of 804 fractures of the shaft of the femur and tibia treated with prolonged immobilisation WatsonJones reported no case of non-union. ${ }^{26}$ Many of these fractures were multiple, comminuted, contaminated, or heavily infected.

Thirdly, the criterion for "success" is the development of a "sound bony union." This end point is by no means always clear, particularly in patients who have had previous surgery with grafting and considerable scarring and distortion of the bone. For "success" to be achieved with electrical treatment several strict conditions must be met. A "cooperative patient" is required to change the anode pad every other day when direct current is used to avoid skin irritation. A plaster of Paris cast is applied "to protect the electrodes," and "non-weight bearing is essential." If weight bearing occurred, "the true effect of the applied constant direct current is not realised ... and irritation may develop around the cathodes or the cathodes may break. ${ }^{14}$ Of necessity the patient's activity is reduced by having to plug in the machine or carry around an electricity supply. Frequent visits to the 